\title{
Butter Mountains, Milk Lakes and Optimal Price Limiters
}

\author{
Ned Corron \\ U.S.Army RDECOM, AMSRD-AMR-WS-ST, Redstone Arsenal, AL 35898, USA \\ Xue-Zhong He \\ School of Finance and Economics, University of Technology, Sydney, PO Box 123 Broadway, \\ NSW 2007, Australia \\ Frank Westerhoff \\ Department of Economics, University of Osnabrueck, Rolandstrasse 8, D-49069 Osnabrueck, \\ Germany
}

Version: April 7, 2005

\begin{abstract}
It is known that simple price limiters may have unexpected consequences in irregular commodity price fluctuations between bull and bear markets and complicated impacts on the size of buffer stocks. In particular, imposing a lower price boundary may lead to a huge buffer stock, e.g. to a "butter mountain" or a "milk lake" and this is a real problem for regulators since storage costs may become impossible to finance over time. The relation between price limiters and the size of buffer stocks is nontrivial and there may exist some optimal price limiters which require only weak market interventions and thus provide a rather inexpensive option to regulate commodity markets. In this paper, we use a simple commodity market model to explore the relation between price limiters and the average growth rate of the buffer stocks. It is found that these optimal price limiter levels are simply the minimum values of unstable periodic orbits of the underlying deterministic system.
\end{abstract}

Keywords: Commodity markets, price stabilization, limiter control, butter mountains and milk lakes.

JEL classification: D84, G18, Q11. 


\section{INTRODUCTION}

Commodity prices are extremely volatile and regularly display severe booms and busts. Many countries thus have experimented with some form of commodity price stabilization scheme in the past (Newbery and Stiglitz (1981)). In particular, attempts have been made to stabilize agricultural commodity markets by means of a commodity buffer stock scheme. The idea of such a scheme is to put a certain amount of output into storage in years in which there is a good harvest, thus increasing the price from what it would have been, and to sell output from the storage in years in which there is a small harvest, thus reducing the price from what it would have been. Although theoretically appealing, these stabilization schemes turned out to be a mixed blessing in reality. Especially in Europe, price guarantees offered by market regulators led to excessive production which had to be taken up by state agencies. The surplus output was stored and periodically amounted in embarrassing phenomena like "butter mountains" and "milk lakes", associated with significant storage costs. It is thus important to understand the relation between price limiters and the growth of buffer stocks, in particular, the optimal price limiters which require only weak market interventions and thus provide a rather inexpensive option to regulate commodity markets, and this is indeed the goal of this paper.

To characterize the nature of commodity price fluctuation, He and Westerhoff (2005) develop a behavioral market model with consumers, producers and heterogeneous speculators. Motivated by recent chaos control literature (e.g. Corron, Pethel and Hopper (2000)), they show that a central authority may stabilize the cyclical behavior of commodity prices by imposing price boundaries. Within this model, we present evidence that the size of buffer stocks depends in a nonlinear way on the imposed price limiters. While most price limiters lead to huge buffer stocks, there also exist some optimal price limiters which may be defendend with rather weak market interventions. We show that these optimal price levels are closely related to the minimum values of unstable periodic orbits of the underlying deterministic system. Hence, if it is politically desirable to stabilize commodity markets, central authorities may have the opportunity to do this without accumulating large and costly inventories.

The rest of our paper is organized as follows. In section 2, we briefly recall the model of He and Westerhoff (2005). In section 3, we we analyze the relationship between price limiters and the growth rate of buffer stocks. The final section concludes the paper.

\section{THE MODEL}

He and Westerhoff (2005) develop a stylized commodity market model with consumers, producers and speculators. ${ }^{1}$ Depending on market conditions, speculators-who play an important role in many commodity markets-either rely on technical or fundamental trading rules to determine their excess demand. The price adjustment is approximated by a log-linear price impact function. The $\log$ price $S$ at time $t+1$ is given as

$$
S_{t+1}=S_{t}+a\left[D_{t}^{M}+W_{t}^{C} D_{t}^{C}+W_{t}^{F} D_{t}^{F}\right]
$$

where $a$ is a positive scaling coefficient to calibrate the price adjustment, $D_{t}^{M}, D_{t}^{C}$ and $D_{t}^{F}$ stand for the excess demand of the real economy, the chartists and the fundamentalists, respectively, at time $t$. The weights of the chartists and the fundamentalists at time $t$ are given as $W_{t}^{C}$ and $W_{t}^{F}$, respectively. According to (1), the price of the commodity increases when there is an excess demand, and vice versa.

Demand and supply of the real economy is described in the reduced way $D_{t}^{M}=m\left(F-S_{t}\right)$. The $\log$ of the long-run equilibrium price is denoted by $F$ and $m$ is a positive parameter. For

\footnotetext{
${ }^{1}$ Their model is inspired by the so-called chartist-fundamentalist approach (e.g. Day and Huang (1990), Kirman (1991), Brock and Hommes (1997), Lux and Marchesi (2000), Farmer and Joshi (2002), Chiarella and He (2003)), which is quite successful in explaining financial market dynamics.
} 
instance, if $S>F$, then the supply of producers exceeds the demand of consumers and, as a result, the excess demand of the real economy is negative.

Speculators are familiar with both technical and fundamental analysis. The excess demand generated by technical analysis is formalized as $D_{t}^{C}=b\left(S_{t}-F\right)$, where $b$ is a positive reaction coefficient. So-called chartists typically believe in bear and bull markets. As long as the price is above (below) its long-run equilibrium value, chartists regard the market as bullish (bearish). Since a further price increase (decrease) is expected, chartists tend to buy (sell) the commodity. On the other hand, fundamental analysis presumes that prices revert toward their long-run equilibrium value. If the price is below (above) its equilibrium value, higher (lower) prices are expected and fundamental analysis favors buying (selling) the commodity. The excess demand generated by fundamental analysis is written as $D_{t}^{F}=c\left(F-S_{t}\right)$. The strength of fundamental analysis depends on the positive reaction coefficient $c$.

Speculators try to exploit bull and bear market situations. But the more the price deviates from its long-run equilibrium value, the greater the speculators perceive the risk that the bull or bear market might collapse. As a result, an increasing number of speculators opt for fundamental trading strategies. The market share of speculators who follow technical analysis is defined as $W_{t}^{C}=1 /\left[1+d\left(F-S_{t}\right)^{2}\right]$. The higher the positive parameter $d$, the faster speculators switch to fundamental analysis as the mispricing increases. The weight of the fundamentalists is, of course, $W_{t}^{F}=1-W_{t}^{C}$.

The above analysis results in the law of motion of the model

$$
S_{t+1}=f\left(S_{t}\right):=S_{t}+a\left[m\left(F-S_{t}\right)-b \frac{F-S_{t}}{1+d\left(F-S_{t}\right)^{2}}+c \frac{d\left(F-S_{t}\right)^{3}}{1+d\left(F-S_{t}\right)^{2}}\right] .
$$

A formal analysis of the properties of this one-dimensional nonlinear map and the impact of price limiters are provided in He and Westerhoff (2005). For example, it is shown that for $a=1, b=4.5, c=1.5, d=1, m=1$ and $F=0$, the map possesses three unstable fixed points $\left(\bar{F}=0, \bar{S}_{ \pm}= \pm 1.18322\right)$ and the model generates intricate (chaotic) price dynamics. In particular, prices fluctuate in a complex way between bull and bear markets, as observed in many commodity markets.

\section{PRice Limiters AND Buffer Stocks}

Control algorithms (e.g., Ott, Grebogi and Yorke (1990), Pyragas (1992), or Parthasarathy and Sinha (1995)) have been developed to stabilize chaotic dynamics. The feasibility of using chaos controllers depends on the complexity and efficiency of the control algorithm. The chaos control process typically requires measurement of the system's state, generation of a control signal, and the application of a control signal to an accessible system parameter. Chaos control of economic system is often regarded with some skepticism since such information is hard to collect. Fortunately, Corron, Pethel and Hopper (2000) present experimental evidence that chaos control can also be accomplished by using simple limiters. This method, which has been analytically and numerically explored by Wagner and Stoop (2000) and Stoop and Wagner (2003), simply restricts the phase space of a trajectory that can be explored. Limiter control is obviously convenient to conduct, even in an economic context. For example, a central authority which imposes price boundaries in commodity markets automatically applies the limiter method. Clearly, price boundaries are a real-life application of limiter control.

To study the emergence of "butter mountains" and "milk lakes", we explore the case of minimum price limiters $S_{\text {min }}$. Hence, (1) becomes

$$
S_{t+1}=S_{t}+a\left[D_{t}^{M}+W_{t}^{C} D_{t}^{C}+W_{t}^{F} D_{t}^{F}+D_{t}^{C A}\right]+\delta_{t}
$$

where $D_{t}^{C A}$ stands for the interventions of the central authority and $\delta_{t}$ is an IID noise term to allow for additional market disturbances not captured by our stylized model. The interventions 
of the central authority just have to offset the excess supply in the market that would otherwise push the price below $S_{\text {min }}$. Hence, it follows from (3) that $D_{t}^{C A}$ is defined as

$$
D_{t}^{C A}=\max \left\{\frac{S_{\min }-S_{t}-\delta_{t}}{a}-D_{t}^{M}-W_{t}^{C} D_{t}^{C}-W_{t}^{F} D_{t}^{F}, \quad 0\right\} .
$$

We are interested in the size or growth rate of the long-term average buffer stock $B_{T}=$ $\frac{1}{T} \sum_{t=1}^{T} D_{t}^{C A}$, which is computed using $T$ observations.
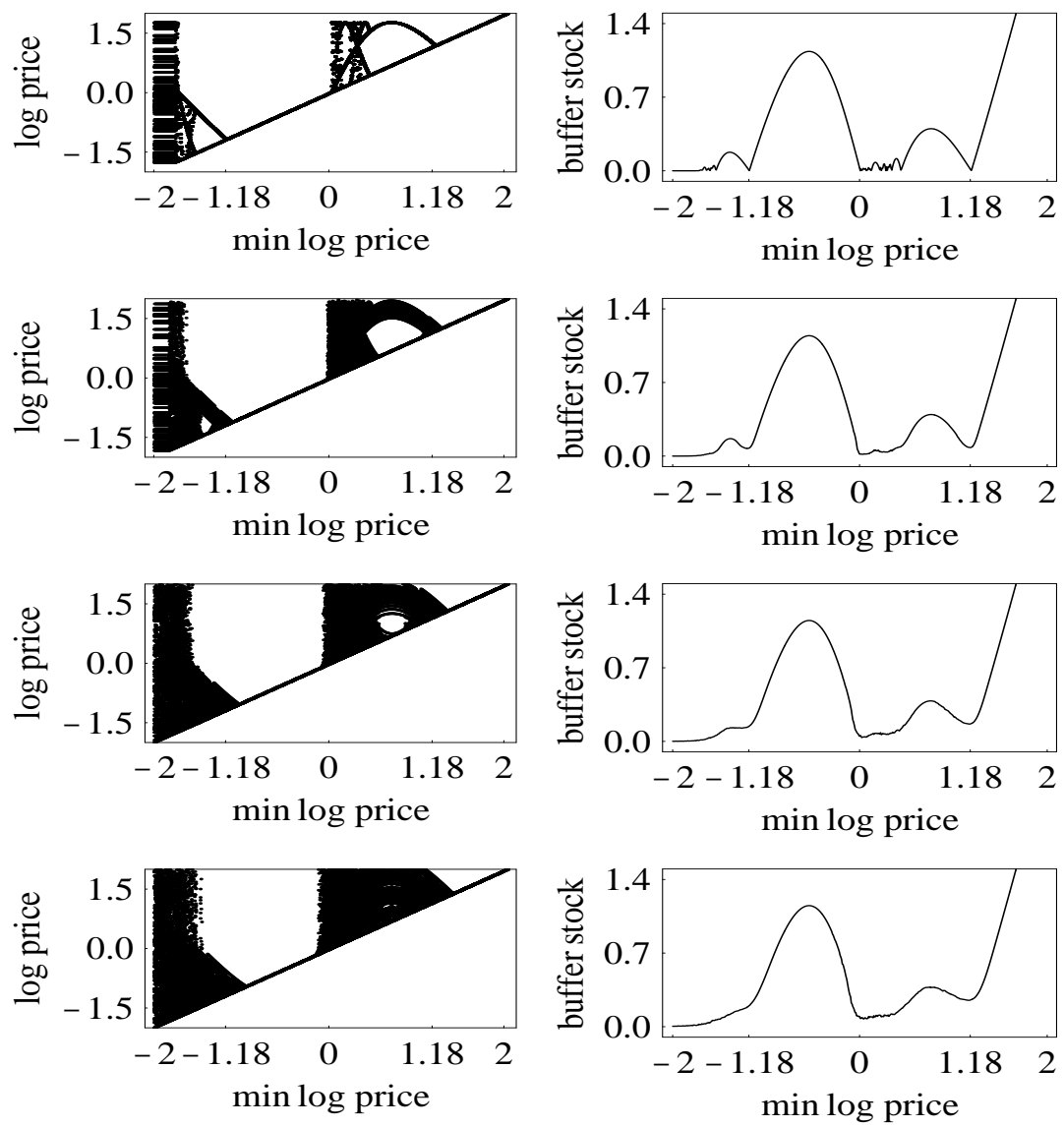

FIGURE 1. The left-hand panels show bifurcation diagrams for lower price boundaries. The price boundaries are increased from -2 to +2 in 500 discrete steps. For each value, 100 observations are plotted. The right hand panels depicts the corresponding growth rate of the buffer stock based on $T=1000$. Parameter setting as in section 2. Noise levels from top to bottom are given as $\delta \sim \mathcal{N}(0, \sigma)$ with $\sigma=0,0.1,0.2,0.3$.

Fig. 1 reveals the impact of price limiters on the evolution of commodity prices (left-hand panels) and the growth rate of the resulting buffer stocks (right-hand panels) with respect to the minimum price limiters and the deterministic case and three noise cases. One can see that the impact of the price limiters on the average growth rate of the buffer stock is neither obvious nor intuitive in both deterministic and noise markets. Defending price boundaries may require substantial interventions of the central authority and thus may lead to huge buffer stocks. Consequently, the storage costs may be quite dramatic. This is consistent with the European experience where embarrassing "butter mountains" and "milk lakes" emerged after guaranteeing farmers minimum prices. Also, the changes of the growth rate of the buffer stock with and without market noise are very similar. However — and this is a surprising message - the growth rate 
of the buffer stock illustrated in Figure 1 depends in a nontrivial way on the established price limiter. For some price limiters the growth rate of the buffer stock is slow (or even negligible).

What is the explanation for this puzzling outcome? In the following discussion, we focus on the deterministic case and offer first an intuitive explanation by looking at the changes of the complex price dynamics resulting from a homoclinic orbit and then a more precise explanation by calculating the growth rate in the buffer stocks under a price limiter. Consequently, we obtain the mechanism in generating the optimal price limiters, under which the growth rates of the buffer stocks are close to zero.

For the given set of parameters, He and Westerhoff (2005) show that simple price limiters may have unexpected consequences - a minimum (maximum) price limiter decreases (increases) the average price. More precisely, map (2) is non-invertible and has three unstable fixed points, the fundamental price $F$ and two non-fundamental prices $S_{ \pm}$(satisfying $S_{-}<F<S_{+}$). The trapping invariant interval of the map consists of two non-invariant overlapping trapping intervals around the two non-fundamental prices. If the price limiter $S_{\min }$ is below the lower bound of the invariant interval, prices evolve as usual and the growth rate of the buffer stocks is zero. The price limiter starts to play a role once it is above the lower bound. As the price limiter increases, the prices are effectively trapped in the invariant interval of the lower nonfundamental price $S_{-}$most of the time and the growth rate of the buffer stocks increases initially and then decreases to zero when the price limiter is given by the lower non-fundamental price. This explains the first small "hump" of the average buffer stock for price limiters below $S_{-}(=$ $-1.18)$ in Fig. 1. A price limiter between $S_{-}$and $F$ prevents the price from constantly dropping below the price limiter under the map. Also, the difference between $S_{\min }$ and $f\left(S_{t}\right)$ increases initially, reaching a local maximum when $S_{\min }$ corresponds to the local minimum point of the function $f$, and then decreases to zero as the price limiter approaches $F$. This explains the big "hump" for the averaged growth rate of the buffer stock between $S_{-}$and $F(=0)$ in Fig. 1 . When the price limiter is above $F=0$, the price limiter traps the price in the invariant interval of $S_{+}$most of the time, leading to the "hump" growth rate of the buffer stocks below $S_{+}(=1.18)$. It becomes zero when $S_{\min }=S_{+}$. However, once $S_{\min }$ is above the upper bound of the invariant interval, the intervention is always needed to support the high lower-price limiter. Consequently, the growth rate of the buffer stock increases and becomes unbounded. This gives us an intuitive explanation on the growth rate behavior of the buffer stocks.

We now give a more precise explanation by calculating the growth rate of the buffer stock (after transients) for a given limiter level. In fact, for the deterministic case, the buffer stock $D_{t}^{C A}$ in (4) reduces to $D_{t}^{C A}=\max \left\{\left[S_{\text {min }}-f\left(S_{t}\right)\right] / a, 0\right\}$. For limiters where $f\left(S_{\text {min }}\right)<S_{\text {min }}$, the limiter is exercised continuously. That is, at every iteration, the limiter must provide additional demand to raise the price to $S_{\text {min }}$. Thus, the growth rate that the buffer stock increases is precisely the scaled difference $\left[S_{\text {min }}-f\left(S_{\min }\right)\right] / a$. For the given set of parameters, the difference is positive for $S_{\min }$ in between -1.18 and 0 and the resulting plot in the upper-left panel in Fig. 2 explains the large hump between -1.18 and 0 and the unbounded growth above 1.18. For limiter values where $f\left(S_{\text {min }}\right)>S_{\text {min }}$ but $f\left(f\left(S_{\text {min }}\right)\right)<S_{\text {min }}$, the limiter is exercised every second iteration, and the growth rate of the buffer stock is given by $\left[S_{\min }-f\left(f\left(S_{\min }\right)\right)\right] /(2 a)$. This is calculated for the $S_{\min }$ satisfying $\left[S_{\min }-f\left(S_{\min }\right)\right] / a \leq 0$. The composite of the above two calculations is shown in the upper-right panel in Fig. 2. Limiting every second iterate explains the bumps just below 1.18 and -1.18. The remaining bumps are explained by limiters acting on still higher order iterates. That is, $f\left(S_{\text {min }}\right)>S_{\text {min }}, f^{(2)}\left(S_{\text {min }}\right)>S_{\text {min }}, \cdots, f^{(N-1)}\left(S_{\text {min }}\right)>S_{\text {min }}$, but $f^{(N)}\left(S_{\text {min }}\right)<S_{\text {min }}$, where $f^{(i)}$ indicates the $i$-th iterate of $f$. In that case the rate of buffer growth is $\left[S_{\min }-f^{(N)}\left(S_{\min }\right)\right] /(a N)$. The composite of the rate for all $N$ up to 3 and 1000 is shown in the down-left and down-right panels, respectively, in Fig. 2. The difference for $N>2$ explains the remaining smaller bumps in the buffer stock growth. This agrees fairly well with the "experimental" result in the top right plot in Fig. 1. 

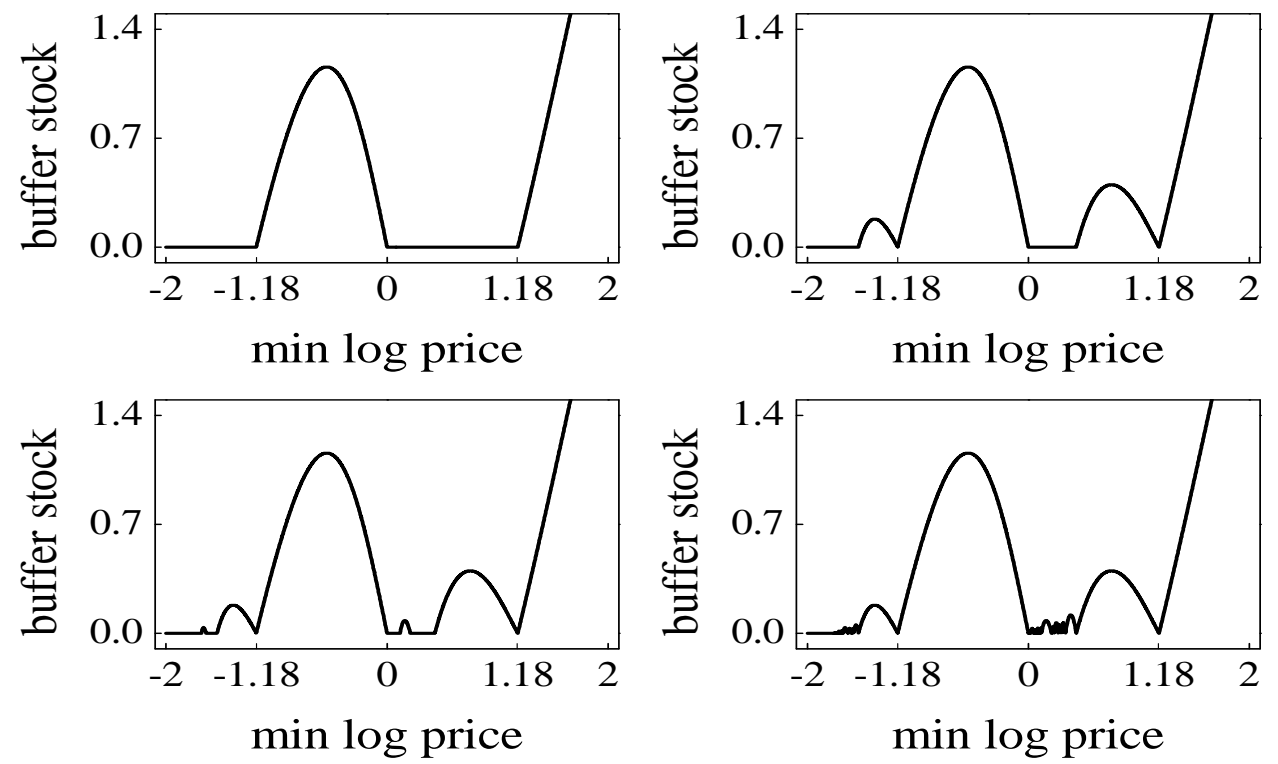

FIGURE 2. The composite of the average growth rate $\left[S_{\min }-\right.$ $\left.f^{(N)}\left(S_{\min }\right)\right] /(a N)$ for all $N$ up to $N=1$ (upper-left), 2 (upper-right), 3 (downleft) and 1,000 (down-right) for 4001 evenly spaced values of $S_{\min }$ in the range $[-2,2]$.

The interesting limiter levels are the points of equality that separate the regions for different $N$. These points are the so-called optimal limiter levels. For example, the boundary for $N=1$ is defined by $f\left(S_{\min }\right)=S_{\text {min }}$, corresponding to the three fixed points $S_{ \pm}, F$. In the absence of noise, the growth rate of the buffer stock is precisely zero for these limiter levels. A similar thing happens for $N=2$. Here, the boundary is defined by $f\left(f\left(S_{\min }\right)\right)=S_{\text {min }}$, corresponding to an unstable period-2 orbit of $f$. The effect of the limiter is to stabilize that periodic orbit without growth in the buffer stock (in the absence of noise). More generally, a boundary for $N$ is given by $f^{(N)}\left(S_{m i n}\right)=S_{m i n}$, corresponding to a period- $N$ orbit. Thus a limiter set precisely to the minimum value of an unstable periodic orbit will stabilize that orbit without growth in the buffer stock. With noise the growth rate of the buffer stock is proportional to the average magnitude of the noise.

Of course, the central authority may not exactly know these buffer stock "minimizing" price boundaries. A central authority thus may experiment for some time with different price boundaries until an acceptable outcome has been approached. The main message of this paper is that if price boundaries are politically desirable, ${ }^{2}$ then a central authority has not necessarily to accumulate large inventories.

\section{Conclusions}

A common concern for price boundaries in commodity markets is the fear of a more or less unlimited growth of the buffer stock. Indeed, notorious surpluses in production of agricultural goods and connected large storage costs have been repeatedly observed in history. Prominent examples include the European "butter mountains" and "milk lakes". In this paper, we explore the impact of price limiters on the evolution of buffer stocks within a simple commodity market model. Price limiters may in fact lead to large buffer stocks. However, the good news is that there also exist some price limiters which stabilize price fluctuations without generating large inventories. Our paper also demonstrates that chaos control algorithms such as the limiter method may help policy makers to improve the regulation of economic systems.

${ }^{2}$ Popular arguments in support of price boundaries are concerned with (i) a country's autarky in agricultural supply, (ii) the regulation of agricultural prices, and (iii) a stabilization of the farmers' income. 


\section{References}

Brock, W. and Hommes, C. (1997): A rational route to randomness. Econometrica, 65, 1059-1095.

Chiarella, C. and He, X.-Z. (2003): Dynamics of beliefs and learning under $a_{L}$ processes the heterogeneous case. Journal of Economic Dynamics and Control, 27, 503-531.

Corron, N., Pethel, S. and Hopper, B. (2000): Controlling chaos with simple limiters. Physical Review Letters, 84, 3835-3838.

Day, R. and Huang, W. (1990): Bulls, bears and market sheep. Journal of Economic Behavior and Organization, 14, 299-329.

De Grauwe, P., Dewachter, H. and Embrechts, M. (1993): Exchange rate theory - chaotic models of foreign exchange markets. Blackwell: Oxford.

Farmer, D. and Joshi, S. (2002): The price dynamics of common trading strategies. Journal of Economic Behavior and Organization, 49, 149-171.

He, X.-Z. and Westerhoff F. (2005): Commodity markets, price limiters and spectulative price dynamics. Journal of Economic Dynamics and Control, in press.

Kirman, A. (1991): Epidemics of opinion and speculative bubbles in financial markets. In: Taylor, M. (Ed.): Money and financial markets. Blackwell: Oxford, 354-368.

Lux, T. and Marchesi, M. (2000): Volatility clustering in financial markets: A micro-simulation of interacting agents. International Journal of Theoretical and Applied Finance, 3, 675-702.

Newbery, D. and Stiglitz, J. (1981): The theory of commodity price stabilization: a study in the economics of risk. Claredon Press: Oxford.

Ott, E., Grebogi, C. and Yorke, J. (1990): Controlling chaos. Physical Review Letters, 64, 1196-1199.

Parthasarathy, S. and Sinha, S. (1995): Controlling chaos in unidimensional maps using constant feedback. Physical Review E, 51, 6239-6242.

Pyragas, K. (1992): Continuous control of chaos by self-controlling feedback. Physical Letters A, 170, 421-428.

Stoop, R. and Wagner, C. (2003): Scaling properties of simple limiter control. Physical Review Letters, 90, 154101-1.

Wagner, C. and Stoop, R. (2000): Optimized chaos control with simple limiters. Physical Review E, 63, 017201. 